\title{
Decays of tensor mesons and the tensor glueball in an effective field approach
}

\author{
F. Giacosa, Th. Gutsche, V. E. Lyubovitskij and Amand Faessler \\ Institut für Theoretische Physik, Universität Tübingen, \\ Auf der Morgenstelle 14,D-72076 Tübingen, Germany
}

(Dated: August 16, 2018)

\begin{abstract}
The strong and electromagnetic decays of the ground-state tensor mesons are studied in an effective field approach. A fit to the well-known experimental data is performed. The decay ratios of the tensor glueball are evaluated and possible candidates are discussed.

PACS numbers: 12.39.Fe, 12.39.Mk, 13.25.Jx, 13.40.Hq

Keywords: Tensor mesons, glueball, effective chiral approach, strong and electromagnetic decays
\end{abstract}

\section{INTRODUCTION}

The experimental state of knowledge about the tensor meson nonet $2^{++}$is well established: the identified resonances $f_{2}(1270), f_{2}^{\prime}(1525), a_{2}(1320)$ and $K_{2}^{*}(1430)$ are listed by the PDG [1] as the lightest tensor states and correspond to the $2^{++}$ground state nonet (two isoscalars, an isotriplet and two isodoublets, respectively). We do not consider here the resonances $f_{2}(1430)$ and $f_{2}(1565)$, omitted from the summary table of [1]. Strong decays of tensor states into two-body modes have been measured by various experiments and the corresponding averages (or fits) reported in Ref. 1] are precise and well determined. Also the two-photon decays of the tensor states $T \rightarrow \gamma \gamma$ are well-known. Among the p-wave quark-antiquark nonets $\left(0^{++}, 1^{++}, 2^{++}\right)$the tensor mesons are the ones which are experimentally best analyzed. Tensor mesons have been studied using different theoretical methods: effective Lagrangian approaches based on vector- and tensor-meson dominance [2]- 5], the naive quark model with possible meson-glueball mixing [6][10], current-algebra approach 11], lattice QCD [12], QCD sum rules [13], the ${ }^{3} P_{0}$ model [14, 15], Chiral Perturbation Theory (ChPT) [16]- 19], Regge model [20], dispersion-relation technique [21], anti-de Sitter QCD [22], etc.

In the present paper we study the decays of the $2^{++}$tensor nonet into two pseudoscalars $T \rightarrow P P$ and into two photons $T \rightarrow \gamma \gamma$ within a chiral approach evaluated at the tree-level. We extend the analysis to the kinematically allowed strong decay modes into a pseudoscalar and a vector meson $T \rightarrow P V$ and to the corresponding radiative decay modes $T \rightarrow P \gamma$.

The basic chiral Lagrangian for the tensor mesons is presented in Refs. [16]-[19]. In particular, in Refs. [16-[17] the contribution of the tensor meson resonances to the low energy coupling constants is evaluated following the idea suggested in the case of pseudoscalar, scalar, vector and axial resonances [23]. In Ref. [19] the attention is focused on the mass spectrum of the tensor mesons. Various decay properties are analyzed in the framework of tensor-meson dominance (TMD) in combination with vector-meson dominance (VDM) 2, 3, 4]. A study on the phenomenological properties of $f_{2}(1270)$ and $f_{2}^{\prime}(1525)$ is performed in $[9]$.

Here we intend to study the decay properties of the full tensor meson nonet by performing a fit of the free parameters in the chiral Lagrangian to the available data 1|. We then turn our attention to some properties of the tensor glueball with an expected mass of about $\sim 2.2 \mathrm{GeV}[24]$. We evaluate the two-pseudoscalar and two-vector decay ratios as following from the simplest form of the interaction Lagrangian and discuss some possible candidates.

In Refs. 25, 26 the strong and radiative decays of the scalar quarkonia nonet supplemented by an intruding glueball state have been evaluated in a chiral approach. The main difficulty of the previous study is a rigorous justification of the chiral approach in the mass region between 1 and $2 \mathrm{GeV}$. At the same time the experimental situation concerning the scalar sector is not yet complete [1]. The tensor meson sector offers a possibility to test a tree-level calculation for p-wave states within a chiral approach in the energy region above $1 \mathrm{GeV}$. The $2^{++}$tensor glueball is expected to have a mass $\sim 2.2 \mathrm{GeV}[24]$ as predicted by lattice calculation. Also, no significant mixing with the ground state quarkonia is expected, as the phenomenological study of [10] confirms. The quarkonia-mixing between the nonstrange $\bar{n} n$ and the strange $\bar{s} s$ component is small, generating an almost ideally mixed nonet. Flavor mixing driven by instantons, which is large in the pseudoscalar sector, unknown but possibly large in the scalar one [26]), does not affect the tensor mesons.

The interest in hadronic resonance physics at an energy scale larger than $1 \mathrm{GeV}$ is growing and the attempts to describe mesonic states in chiral approaches become more numerous. For instance, in [27] the radiative $P V$ decays of axial states (with mass around $1.3 \mathrm{GeV}$ ) were evaluated. At higher energy scales the calculation of higher order corrections or possible final state interaction poses a problem to a chiral approach. However, the chiral Lagrangian with tree-level evaluations presents a useful phenomenological tool for the description of high-lying resonances.

The paper is organized as follows. In the next section we describe the chiral Lagrangian for the $P P$ and $\gamma \gamma$ decays 
of tensor mesons. In Sec.3 we perform a fit to the known experimental widths and we discuss the $\gamma \gamma, P V$ and $P \gamma$ transitions. In Sec. 4 we consider the decays of the unmixed tensor glueball with a mass around $2.2 \mathrm{GeV}$ and discuss some physical resonances. Finally, in Sec.5 we give our conclusions.

\section{EFFECTIVE LAGRANGIAN FOR DECAYS OF TENSOR MESONS}

The effective Lagrangian describing the strong and electromagnetic decays of tensor mesons $f_{2}(1270), f_{2}^{\prime}(1525)$, $a_{2}(1320)$ and $K_{2}^{*}(1430)$ is given by (see detailed discussion in Refs. [3, 16, 17, 18, 19])

$$
\begin{aligned}
\mathcal{L}_{\text {eff }}^{T} & =\frac{F^{2}}{4}\left\langle D_{\mu} U D^{\mu} U^{\dagger}+\chi_{+}\right\rangle-\frac{1}{4}\left\langle\mathcal{T}_{\mu \nu} D^{\mu \nu ; \rho \sigma} \mathcal{T}_{\rho \sigma}\right\rangle-\frac{1}{4}\left\langle\mathcal{V}_{\mu \nu} \mathcal{V}^{\mu \nu}-2 M_{V}^{2} \mathcal{V}_{\mu} \mathcal{V}^{\mu}\right\rangle+\mathcal{L}_{\text {mix }}^{P}+\mathcal{L}_{\text {mix }}^{T} \\
& +c_{T P P}^{8}\left\langle\mathcal{T}_{\mu \nu}^{o c t e t} \Theta_{P}^{\mu \nu}\right\rangle+\frac{c_{T P P}^{0}}{\sqrt{3}} T_{\mu \nu}^{0}\left\langle\Theta_{P}^{\mu \nu}\right\rangle+c_{T \gamma \gamma}\left\langle\mathcal{T}_{\mu \nu} \Theta_{\gamma}^{\mu \nu}\right\rangle \\
& +i c_{T P V}\left\langle\mathcal{T}^{[\mu \nu] \alpha}\left[\widetilde{\mathcal{V}}_{\mu \nu}, \partial_{\alpha} \mathcal{P}\right]\right\rangle+i c_{T P \gamma}\left\langle\mathcal{T}^{[\mu \nu] \alpha}\left[Q \widetilde{F}_{\mu \nu}, \partial_{\alpha} \mathcal{P}\right]\right\rangle,
\end{aligned}
$$

where the nonets of tensor, vector and pseudoscalar mesons are defined as

$$
\mathcal{T}_{\mu \nu}=\frac{1}{\sqrt{2}} \sum_{i=0}^{8} T_{\mu \nu}^{i} \lambda_{i}=\mathcal{T}_{\mu \nu}^{\text {octet }}+T_{\mu \nu}^{0} \frac{\lambda_{0}}{\sqrt{2}}, \quad \mathcal{V}_{\mu}=\frac{1}{\sqrt{2}} \sum_{i=0}^{8} V_{\mu}^{i} \lambda_{i}, \quad \mathcal{P}=\frac{1}{\sqrt{2}} \sum_{i=0}^{8} P^{i} \lambda_{i} .
$$

Here and as follows the symbols $\langle\ldots\rangle,[\ldots]$ and $\{\ldots\}$ denote the trace over flavor matrices, the commutator and anticommutator, respectively.

The constants $c_{T P P}^{8}, c_{T P P}^{0}, c_{T \gamma \gamma}, c_{T P V}$ and $c_{T P \gamma}$ define the coupling of tensor fields to photons, pseudoscalar and vector mesons. We indicate the strong decays of the octet (coupling $c_{T P P}^{8}$ ) and the singlet (coupling $\left.c_{T P P}^{0}\right)$ states separately. However, we do not expect a large violation of the condition $c_{T P P}^{8}=c_{T P P}^{0}$ predicted in the large $N_{c}$ limit.

We use the standard notations for the basic blocks of the ChPT Lagrangian [28, 29]: $U=u^{2}=\exp (i \mathcal{P} \sqrt{2} / F)$ is the chiral field collecting pseudoscalar fields in the exponential parametrization, $D_{\mu}$ denotes the chiral and gaugeinvariant derivative, $u_{\mu}=i u^{\dagger} D_{\mu} U u^{\dagger}$ is the chiral field, $\chi_{ \pm}=u^{\dagger} \chi u^{\dagger} \pm u \chi^{\dagger} u, \chi=2 B(s+i p), s=\mathcal{M}+\ldots, \mathcal{M}=$ $\operatorname{diag}\left\{\hat{m}, \hat{m}, m_{s}\right\}$ is the mass matrix of current quarks (we restrict to the isospin symmetry limit with $m_{u}=m_{d}=\hat{m}$ ), $B$ is the quark vacuum condensate parameter and $F$ is the pseudoscalar meson decay constant.

The term $D^{\mu \nu ; \rho \sigma}$ is the inverse propagator of the tensor fields and is given by [16]:

$$
\begin{aligned}
D^{\mu \nu ; \rho \sigma} & =\left(\square+M_{\mathcal{T}}^{2}\right)\left[\frac{1}{2}\left(g^{\mu \rho} g^{\nu \sigma}+g^{\nu \rho} g^{\mu \sigma}\right)-g^{\mu \nu} g^{\rho \sigma}\right]+g^{\rho \sigma} \partial^{\mu} \partial^{\nu}+g^{\mu \nu} \partial^{\rho} \partial^{\sigma} \\
& -\frac{1}{2}\left(g^{\nu \sigma} \partial^{\mu} \partial^{\rho}+g^{\nu \rho} \partial^{\mu} \partial^{\sigma}+g^{\mu \sigma} \partial^{\nu} \partial^{\rho}+g^{\mu \rho} \partial^{\nu} \partial^{\sigma}\right),
\end{aligned}
$$

where $M_{\mathcal{T}}$ is the tensor nonet mass.

The tensors $\Theta_{P}^{\mu \nu}$ and $\Theta_{\gamma}^{\mu \nu}$ are constructed with the use of chiral and electromagnetic fields:

$$
\begin{aligned}
& \Theta_{P}^{\mu \nu}=\frac{F^{2}}{4}\left\{u^{\mu}, u^{\nu}\right\}-\frac{F^{2}}{2} g^{\mu \nu}\left(u^{\sigma} u_{\sigma}+\chi_{+}\right), \\
& \Theta_{\gamma}^{\mu \nu}=F_{\alpha}^{+\mu} F^{+\alpha \nu}+\frac{1}{4} g^{\mu \nu} F^{+\rho \sigma} F_{\rho \sigma}^{+},
\end{aligned}
$$

where $F_{\mu \nu}^{+}=u^{\dagger} F_{\mu \nu} Q u+u F_{\mu \nu} Q u^{\dagger}, \quad F_{\mu \nu}=\partial_{\mu} A_{\nu}-\partial_{\nu} A_{\mu}$ is the stress tensor of the electromagnetic field and $Q=e \operatorname{diag}\{2 / 3,-1 / 3,-1 / 3\}$ is the quark charge matrix.

The tensors $\mathcal{V}^{\mu \nu}, \mathcal{T}^{[\mu \nu] \alpha}$ and the dual tensors $\widetilde{\mathcal{V}}_{\mu \nu}, \widetilde{F}_{\mu \nu}$ are defined as

$$
\begin{aligned}
\mathcal{V}^{\mu \nu} & =\partial^{\mu} \mathcal{V}^{\nu}-\partial^{\nu} \mathcal{V}^{\mu}, & \mathcal{T}^{[\mu \nu] \alpha} & =\partial^{\mu} \mathcal{T}^{\nu \alpha}-\partial^{\nu} \mathcal{T}^{\mu \alpha}, \\
\widetilde{\mathcal{V}}_{\mu \nu} & =\frac{1}{2} \varepsilon_{\mu \nu \rho \sigma} \mathcal{V}^{\rho \sigma}, & \widetilde{F}_{\mu \nu} & =\frac{1}{2} \varepsilon_{\mu \nu \rho \sigma} F^{\rho \sigma}
\end{aligned}
$$

We refer to [26, 30, 31] for the discussion of the term $\mathcal{L}_{\text {mix }}^{P}$ in the Lagrangian, which contains the pseudoscalar masses and the pseudoscalar mixing. As a result, the physical states are expressed in terms of the pseudoscalar octet and singlet states $P^{0}$ and $P^{8}$ :

$$
\eta=P^{8} \cos \theta_{P}-P^{0} \sin \theta_{P}, \quad \eta^{\prime}=P^{8} \sin \theta_{P}+P^{0} \cos \theta_{P},
$$


where the pseudoscalar mixing angle reads $\theta_{P}=-9.95^{\circ}$ at tree-level [26].

Here we restrict to the tree-level evaluation, we therefore consistently use the corresponding tree-level result of $\theta_{P}=-9.95^{\circ}$. In the present approach we do not include the neutral pion when considering mixing in the pseudoscalar sector, because we work in the isospin limit. This mixing is small and can be safely neglected when studying the decay of tensor resonances into two pseudoscalars. Similarly, for all pseudoscalar mesons we use the unified leptonic decay constant $F$, which is identified with the pion decay constant $F=F_{\pi}=92.4 \mathrm{MeV}$. A more accurate analysis including higher orders should use the individual couplings of the pseudoscalar mesons (for a detailed discussion see Refs. [28, 29]).

The splitting of the nonet masses and the singlet-octet mixing are generated by the Lagrangian $\mathcal{L}_{\text {mix }}^{T}$ (see 19 , 32]). As a result the physical isoscalar tensor states $f_{2} \equiv f_{2}(1270)$ and $f_{2}^{\prime} \equiv f_{2}^{\prime}(1525)$ are expressed in terms of the octet $T^{8}$ and singlet $T^{0}$ components by the tensor mixing angle $\theta_{T}$ (covariant indices $\mu \nu$ are suppressed):

$$
f_{2}=T^{0} \cos \theta_{T}+T^{8} \sin \theta_{T}, \quad f_{2}^{\prime}=-T^{0} \sin \theta_{T}+T^{8} \cos \theta_{T} .
$$

The expressions for the two-pseudoscalar decays are derived from the Lagrangian of Eq. (1) and are listed in the Appendix A. Note, that these decays have been discussed previously in Ref. 6]. We also report the analytical expressions for the decay rates of the isovector $a_{2}$ and isodoublet $K_{2}^{*}$ states in the Appendix A.

The physical vector mesons $\omega$ and $\phi$ are given by (covariant index $\mu$ understood):

$$
\omega=V^{0} \cos \theta_{V}+V^{8} \sin \theta_{V}, \phi=-V^{0} \sin \theta_{V}+V^{8} \cos \theta_{V} .
$$

The vector meson mixing angle $\theta_{V}$ is found to be $39^{\circ}$ in 32 (the value we use), not far from the ideal mixing angle of $\theta_{V_{I}}=35.3^{\circ}$; this in turn means that $\omega \sim \sqrt{1 / 2}(\bar{u} u+\bar{d} d) \equiv \bar{n} n$ and $\phi \sim \bar{s} s$. Here we describe vector mesons in terms of vector fields. Alternatively, vector mesons can be represented in terms of antisymmetric tensor fields which is most convenient for constructing chiral Largrangians involving vector mesons and their couplings to pseudoscalar mesons, baryons and photons [29, 30, 32, 33].

\section{RESULTS}

\section{A. Two-pseudoscalar decays}

In this section we perform a fit to the two-body pseudoscalar $P P$ decays of tensor mesons, in particular the $\pi \pi$, $\bar{K} K, \eta \eta$ modes for $f_{2} \equiv f_{2}(1270)$ and $f_{2}^{\prime} \equiv f_{2}^{\prime}(1525)$, the $\bar{K} K, \eta \pi, \eta^{\prime} \pi$ modes for $a_{2}(1370)$ and the $\bar{K} K$ mode for $K_{2}^{*}(1430)$ (the $K \eta$ mode is not considered in the fit, see below). The corresponding experimental results, as deduced from [1], are reported in Table 1. In case of a asymmetric error the largest value is used. The measured partial decay width $\Gamma_{K_{2}^{*} \rightarrow K \eta} /\left(\Gamma_{K_{2}^{*}}\right)_{t o t}=1.5_{-1.0}^{+3.4} \times 10^{-3}$ does not allow this procedure, we therefore do not include it in the fit directly but compare later.

For the partial widths of the various states listed in Table 1 we use the full widths reported in [1]. For $K_{2}^{*}(1430)$ an average over the neutral and the charged $K_{2}^{*}(1430)$ widths is performed, finding: $\left(\Gamma_{K_{2}^{*}}\right)_{t o t}=103.75 \pm 3.85 \mathrm{MeV}$.

The free parameters entering in the expressions for the $T \rightarrow P P$ decays are the two decay strengths $c_{T P P}^{8}$ and $c_{T P P}^{0}$ introduced in Eq. (1) and the tensor mixing angle $\theta_{T}$ (see Eq. (7)). The tensor meson masses are taken from Ref. [1]: $M_{f_{2}}=1275.4 \pm 1.2 \mathrm{MeV}, M_{a_{2}}=1318.3 \pm 0.6 \mathrm{MeV}, M_{K_{2}^{*}}=1429 \pm 1.4 \mathrm{MeV}$ (average over the neutral and the charged states) and $M_{f_{2}^{\prime}}=1525 \pm 5 \mathrm{MeV}$.

A $\chi^{2}$ minimum is obtained for the following values:

$$
c_{T P P}^{8}=0.0353 \mathrm{GeV}, c_{T P P}^{0}=0.0410 \mathrm{GeV}, \theta_{T}=28.78^{\circ} \text { with } \chi_{\text {tot }}^{2}=18.496 .
$$

The singlet-octet ratio $y_{T P P}=c_{T P P}^{0} / c_{T P P}^{8}=1.161$ is close to unity, as expected from the strong coupling limit. The tensor mixing angle $\theta_{T}=28.78^{\circ}$ is not far from the ideal mixing angle $\theta_{T_{I}}=35.3^{\circ}$. In the chiral study of 32 ] the value $\theta_{T}=32^{\circ}$ is obtained, while in the phenomenological study of $[9]$ the slightly smaller tensor angle $\theta_{T}=28.17^{\circ}$ is found.

The mixing matrices connecting the physical states $f_{2}, f_{2}^{\prime}$ to the bare ones, $T^{0}$ and $T^{8}$, or to $\bar{n} n \equiv 1 / \sqrt{2}(\bar{u} u+\bar{d} d)$ and $\bar{s} s$ read explicitly:

$$
\left(\begin{array}{l}
f_{2} \\
f_{2}^{\prime}
\end{array}\right)=\left(\begin{array}{cc}
0.876 & 0.481 \\
-0.481 & 0.876
\end{array}\right)\left(\begin{array}{l}
T^{0} \\
T^{8}
\end{array}\right)=\left(\begin{array}{cc}
0.993 & 0.113 \\
0.113 & -0.993
\end{array}\right)\left(\begin{array}{c}
\bar{n} n \\
\bar{s} s
\end{array}\right) .
$$

The physical states are very close to pure $\bar{n} n$ and $\bar{s} s$ configurations, which is particularly evident from the small $f_{2}^{\prime} \rightarrow \pi \pi$ partial decay widths. Contrary to the pseudoscalar sector and perhaps to the scalar one [26, 34] a large flavor mixing in the tensor nonet is not expected.

The fit results are summarized in Table 1. 
Table 1. Decay properties of tensor mesons.

\begin{tabular}{|l|c|c|l|}
\hline Mode & Experiment $(\mathrm{MeV})$ & Theory $(\mathrm{MeV})$ & $\chi_{i}^{2}$ \\
\hline$\Gamma_{f_{2} \rightarrow \pi \pi}$ & $157.0 \pm 7.6$ & 153.51 & 0.210 \\
\hline$\Gamma_{f_{2} \rightarrow \bar{K} K}$ & $8.5 \pm 0.9$ & 9.15 & 0.526 \\
\hline$\Gamma_{f_{2} \rightarrow \eta \eta}$ & $0.83 \pm 0.20$ & 0.80 & 0.023 \\
\hline$\Gamma_{f_{2}^{\prime} \rightarrow \pi \pi}$ & $0.60 \pm 0.16$ & 0.55 & 0.102 \\
\hline$\Gamma_{f_{2}^{\prime} \rightarrow \bar{K} K}$ & $64.8 \pm 7.6$ & 41.64 & 9.288 \\
\hline$\Gamma_{f_{2}^{\prime} \rightarrow \eta \eta}$ & $7.5 \pm 2.9$ & 6.49 & 0.121 \\
\hline$\Gamma_{a_{2} \rightarrow \bar{K} K}$ & $5.2 \pm 1.1$ & 6.64 & 1.716 \\
\hline$\Gamma_{a_{2} \rightarrow \eta \pi}$ & $15.5 \pm 2.0$ & 18.42 & 2.134 \\
\hline$\Gamma_{a_{2} \rightarrow \eta^{\prime} \pi}$ & $0.57 \pm 0.12$ & 0.80 & 3.652 \\
\hline$\Gamma_{K_{2}^{*} \rightarrow \bar{K} K}$ & $51.8 \pm 3.2$ & 49.08 & 0.722 \\
\hline$\chi_{\text {tot }}^{2}$ & - & - & 18.496 \\
\hline
\end{tabular}

The description of the experimental data $\left(\chi_{\text {tot }}^{2} / N=1.85\right)$ is good. The largest contribution to $\chi^{2}$ comes from an underestimate of the $\bar{K} K$ mode for the $f_{2}^{\prime}(1525)$ resonance.

The theoretical prediction for the branching ratio $K_{2}^{*} \rightarrow K \eta$ is

$$
\Gamma_{K_{2}^{*} \rightarrow K \eta} /\left(\Gamma_{K_{2}^{*}}\right)_{t o t}=3.93 \times 10^{-3}
$$

which within errors is in agreement with the corresponding experimental value of $1.5_{-1.0}^{+3.4} \times 10^{-3}$.

\section{B. Two-photon decays}

We now turn to $\gamma \gamma$ decays of the isoscalar and neutral isovector tensor states. The ratios $\Gamma_{f_{2}^{\prime} \rightarrow \gamma \gamma} / \Gamma_{f_{2} \rightarrow \gamma \gamma}$ and $\Gamma_{a_{2} \rightarrow \gamma \gamma} / \Gamma_{f_{2} \rightarrow \gamma \gamma}$ do not depend on the strength $c_{T \gamma \gamma}$ in (11) and numerically read

$$
\Gamma_{f_{2}^{\prime} \rightarrow \gamma \gamma} / \Gamma_{f_{2} \rightarrow \gamma \gamma}=0.046, \quad \Gamma_{a_{2} \rightarrow \gamma \gamma} / \Gamma_{f_{2} \rightarrow \gamma \gamma}=0.378
$$

where the tensor mixing angle $\theta_{T}=28.17^{\circ}$ is used as fixed by the fit (see (9)). The corresponding experimental values are 1]:

$$
\begin{aligned}
& \left(\Gamma_{f_{2}^{\prime} \rightarrow \gamma \gamma} / \Gamma_{f_{2} \rightarrow \gamma \gamma}\right)_{\exp }=0.031 \pm 0.010 \\
& \left(\Gamma_{a_{2} \rightarrow \gamma \gamma} / \Gamma_{f_{2} \rightarrow \gamma \gamma}\right)_{\exp }=0.383 \pm 0.057
\end{aligned}
$$

Again, the small value of $\Gamma_{f_{2}^{\prime} \rightarrow \gamma \gamma} / \Gamma_{f_{2} \rightarrow \gamma \gamma}$ is extremely sensitive to the precise value of the tensor mixing angle.

In [3, 4, 5] a method is used to fix the strength of the two-photon decays: the tensor meson dominance (TMD) hypothesis allows to determine the coupling of tensor mesons to vector mesons and the subsequent application of vector meson dominance (VMD) allows to deduce the two-photon decay rates. In the present work we do not intend to perform a systematic study of the $T V V$ coupling (and therefore of VMD). The decay into two vectors is generally not kinematically allowed for a ground state tensor meson. The presence of a $4 \pi$ decay mode for the state $f_{2}$ is indeed an indication of a contribution of virtual vector mesons, which then decay into pions. The calculation of such contributions is possible by taking properly into account the finite widths of the resonances and the corresponding virtual states, but, although being an interesting subject, will not be analyzed in the present work. As indicated in [3], the results obtained by applying VMD compare only moderately to the data.

\section{Vector-Pseudoscalar decays}

The isovector state $a_{2}$ decays into $\rho \pi\left(\bar{K} K^{*}(892)\right.$ is not kinematically allowed), the isodoublets $K_{2}^{*}$ into $\bar{K} K^{*}(892)$, $K \rho$ and $K \omega$. By fixing the strength parameter $c_{T P V}$ to reproduce the decay rate of $a_{2} \rightarrow \rho \pi$ we can predict the other three rates (see Table 2). The presented lowest-order results for the decay of $K_{2}^{*}(1430)$, depending only on one free parameter and on flavor symmetry, are rather good. 
Table 2. $T \rightarrow P V$ decays

\begin{tabular}{|l|l|l|}
\hline Quantity & Experiment $(\mathrm{MeV})$ & Theory $(\mathrm{MeV})$ \\
\hline$\Gamma_{a_{2} \rightarrow \pi \rho}$ & $75.0 \pm 6.4$ & 75.0 (fixed) \\
$\Gamma_{K_{2}^{*} \rightarrow \pi K^{*}(892)}$ & $24.5 \pm 1.4$ & 28.97 \\
$\Gamma_{K_{2}^{*} \rightarrow K \rho}$ & $8.6 \pm 1.0$ & 7.40 \\
$\Gamma_{K_{2}^{*} \rightarrow K \omega}$ & $2.86 \pm 0.87$ & 2.64 \\
\hline
\end{tabular}

\section{Pseudoscalar-photon decays}

The decay rates $a_{2}^{ \pm} \rightarrow \pi^{ \pm} \gamma$ and $K_{2}^{* \pm} \rightarrow K^{ \pm} \gamma$ depend on the coupling constant $c_{T P \gamma}$, but their ratio does not and reads:

$$
\frac{\Gamma_{K_{2}^{* \pm} \rightarrow K^{ \pm} \gamma}}{\Gamma_{a_{2}^{ \pm} \rightarrow \pi^{ \pm} \gamma}}=0.83,
$$

which we compare to the experimental value of

$$
\left(\frac{\Gamma_{K_{2}^{* \pm} \rightarrow K^{ \pm} \gamma}}{\Gamma_{a_{2}^{ \pm} \rightarrow \pi^{ \pm} \gamma}}\right)_{\exp }=\frac{0.236 \pm 0.056}{0.287 \pm 0.047}=0.82 \pm 0.29,
$$

which is in good agreement.

As already indicated in the previous subsection, we do not intend to evaluate the radiative decays via vectormeson-dominance. Note, however, that the final states $P V$ are in a $d$-wave, thus implying a fifth power of the relative momentum (see Appendix A) and therefore the kinematical contribution dominates. A naive application of VMD leads therefore to an overestimate of the $P \gamma$-width.

\section{DECAYS OF TENSOR GLUEBALL}

\section{A. $\quad P P$ and $V V$ decay ratios}

According to Lattice QCD the lightest glueball has quantum numbers $J^{P C}=0^{++}$and a mass of about $1.5 \mathrm{GeV}[24]$, which likely mixes with the nearby quarkonia states generating the three scalar-isoscalar resonances $f_{0}(1370), f_{0}(1500)$ and $f_{0}(1710)$. This mixing scenario, although not unique (see 34, 35] and Refs. therein), has been analyzed in various ways: at a composite level in the quantum mechanical studies of [7, 36, 37], in the Lattice study of [38] and by means of a chiral approach [25, 26]. Also, attempts at a microscopic quark-gluon level as in [39] (and Refs. therein) have been performed.

Lattice QCD sets the tensor glueball mass around $2.2 \mathrm{GeV}[24$. A significant mixing with the tensor ground state mesons analyzed in the previous section can be excluded due to the large mass difference (see the study of [10]). On the other hand, a mixing with excited isoscalar-tensor quarkonia states lying in the same energy region is possible, but unfortunately very difficult to deduce: the experimental informations are scarce and the application of theoretical methods is only partially reliable.

In the following we evaluate the two-pseudoscalar and two-vector decay ratios for a hypothetical flavour-blind composite state with a mass of about $\sim 2.2 \mathrm{GeV}$, where glueball-quarkonia mixing is neglected: although one should be aware of such an eventuality, an analysis of mixing requires a certain amount of data to deduce the wave-function contributions. Indeed, the possibility of a small glueball-quarkonia mixing in the tensor sector can be conceived, as we discuss in the next subsection.

Neglecting phase space and flavor symmetry breaking, the two-pseudoscalar ratios for a flavor-blind tensor glueball $G_{\mu \nu}$ follow the well-known pattern:

$$
\pi \pi: \bar{K} K: \eta \eta: \eta \eta^{\prime}: \eta^{\prime} \eta^{\prime}=3: 4: 1: 0: 1 .
$$

By introducing the glueball as a flavor-blind composite field we write down the effective Lagrangian describing its decays into two photons and two vector mesons:

$$
\mathcal{L}_{\text {eff }}^{G}=c_{G P P} G_{\mu \nu}\left\langle\Theta_{P}^{\mu \nu}\right\rangle+c_{G V V} G_{\mu \nu}\left\langle\mathcal{V}^{\mu} \mathcal{V}^{\nu}\right\rangle
$$


Fixing the tensor glueball mass as $M_{G_{2}}=2.2 \mathrm{GeV}$ (in accord with Lattice) the $P P$-ratios become:

$$
\pi \pi: \bar{K} K: \eta \eta: \eta \eta^{\prime}: \eta^{\prime} \eta^{\prime}=1: 0.79: 0.17: 0: 0.001 .
$$

Compared to the flavor ratios of (16), here the $\pi \pi$ is enhanced and $\eta^{\prime} \eta^{\prime}$ is highly suppressed because of available phase space. The $\eta \eta^{\prime}$ is still zero, since no flavor-breaking term is present in (17).

Similarly, for the two-vector decay ratios one has due to flavor symmetry considerations

$$
\rho \rho: \bar{K}^{*} K^{*}: \omega \omega: \omega \phi: \phi \phi=3: 4: 1: 0: 1 .
$$

Inclusion of the phase space correction changes the $V V$-ratios (19):

$$
\rho \rho: \bar{K}^{*} K^{*}: \omega \omega: \omega \phi: \phi \phi=1: 0.84: 0.32: 0: 0.11 .
$$

Note, in section $11 \mathrm{~A}$ we did not consider the decays of the ground-state tensor mesons into pair of vector mesons, because such a decay is generally not kinematically allowed. This kind of approach has been performed in [3, 4], where TMD is employed to deduce the $T V V$ interaction. This approach is the starting point of the analysis of [5], where the two-vector and the radiative decays of a gluonic state with a mass $M \gtrsim 2 \mathrm{GeV}$ are studied. Their results are similar to (20), apart from the $\omega \phi$ mode, large in [5] and zero in our approach : the $\omega \phi$ mode is zero (independent on the choice of the vector mixing angle $\theta_{V}$ ), because a flavor singlet cannot decay into a singlet and an octet (or a mixture of those) because of $U(3)$ flavor-symmetry. This phenomenon is completely analogous to the predicted zero decay mode of a scalar (or a tensor) glueball into $\eta \eta^{\prime}$. In [26] a possible $\eta \eta^{\prime}$ decay of the (unmixed) scalar glueball is generated by a $U(3)$ flavor breaking contribution. We also refer to the work of [20], where the flavor coefficients for the tensor glueball into two vectors are reported (leading-order results in the flavor symmetry limit): the $\eta \eta^{\prime}$ and the $\omega \phi$ modes are forbidden because of $U(3)$ flavor-symmetry. A breaking of this symmetry is possible, but then also the other modes are affected [20, 26]. For completeness we consider such a possibility of the $U(3) \rightarrow S U(3)$ breaking when the nonet of vector mesons is splitted into octet and singlet states. Then we have different couplings of the tensor glueball to octet $\left(c_{G V V}^{8}\right)$ and singlet $\left(c_{G V V}^{0}\right)$ states:

$$
\mathcal{L}_{\text {eff }}^{G V V}=c_{G V V}^{8} G_{\mu \nu}\left\langle\mathcal{V}^{\mu \text { octet }} \mathcal{V}^{\nu \text { octet }}\right\rangle+c_{G V V}^{0} G_{\mu \nu} V^{\mu 0} V^{\nu 0}
$$

The decay amplitudes are reported in the Table 8 in the Appendix: the $\omega \phi$ mode is now allowed and the corresponding amplitude is proportional to $\left[1-c_{G V V}^{0} / c_{G V V}^{8}\right]$, therefore is a higher order correction to the large $N_{c}$ limit with $c_{G V V}^{0}=c_{G V V}^{8}$. No large violation from this limit is expected, as the two-pseudoscalar decay modes confirmed (resulting in $y_{T P P}=c_{T P P}^{0} / c_{T P P}^{8}=1.161$, see Sec.II). For $c_{G V V}^{0} / c_{G V V}^{8} \leq 1.56$ we still have a small $\omega \phi$ mode with $\omega \phi / \rho \rho \leq 0.1$. For $c_{G V V}^{0} / c_{G V V}^{8}=1.56$ the ratios read

$$
\rho \rho: \bar{K}^{*} K^{*}: \omega \omega: \omega \phi: \phi \phi=1: 0.84: 0.58: 0.1: 0.16,
$$

therefore still in qualitative agreement with [20].

In order to get $\omega \phi / \rho \rho \approx 1$ as in [5] we have to increase the value of the ratio $c_{G V V}^{0} / c_{G V V}^{8}$ up to $\approx 2.79$, implying a large (and unnatural) difference between the octet and the singlet decay parameters. For $c_{G V V}^{0} / c_{G V V}^{8}=2.79$ we have

$$
\rho \rho: \bar{K}^{*} K^{*}: \omega \omega: \omega \phi: \phi \phi=1: 0.84: 1.41: 1.00: 0.32 .
$$

The $\omega \omega$ mode has also been modified, being very large in this scenario.

In the end, a strong $\omega \phi$ mode is possible only by introducing a consistent violation from the large $N_{c}$ limit, corresponding to a flavor-undemocratic tensor glueball decay. Although such an eventuality cannot be excluded (see [26] for the discussion in the scalar sector, where however the "undemocracy" is a result of a flavor-symmetry breaking term, affecting also the kaonic decay modes, and not the octet-singlet splitting) is at the present state of knowledge not verifiable and in disagreement with other results, where large $N_{c}$ is, although broken, still approximately valid.

\section{B. Discussion of $f_{J}(2220)$ as a tensor-glueball candidate}

Limiting our study to the mass region $M \gtrsim 2 \mathrm{GeV}$, the following isoscalar tensor states are listed in [1]: $f_{2}(2010)$, $f_{2}(2150), f_{J}(2220)\left(J=0\right.$ or 2 , which still needs to be settled), $f_{2}(2300)$ and $f_{2}(2340)$. 
In [5] the decays of a flavor-blind tensor glueball have been evaluated for $f_{2}(2010)$ and $f_{2}(2300)$. In [20] the analysis of Regge-trajectories for the tensor states leads to the interpretation of $f_{2}(2010)$ and the $f_{2}(2300)$ as dominant $\bar{s} s$ states (note, that a different naming scheme from [1] is used in 20]). According to 20] all the isoscalar tensor states, with the exception of $f_{J}(2220)$ and a broad tensor state around $2 \mathrm{GeV}$ (not listed in 1]), can be interpreted as quarkonia. The broad state $(\Gamma \sim 500 \mathrm{MeV}$ ) around $2 \mathrm{GeV}$ (mass between 1.7 and $2.5 \mathrm{GeV}$ ) found in the analysis of [20] and denoted as $f_{2}(2000)$, but not listed in the compilation of [1], is interpreted as the tensor glueball (see also [40]). The debated issue of the full width of the glueball in the scalar sector [7, 25, 26, 36, 38] is one of the main questions in the tensor sector as well. Another possible candidate for the tensor glueball is the very narrow state $f_{J}(2220)\left(\Gamma_{t o t}=23_{-7}^{+8} \mathrm{MeV}\right)$, also not lying on the Regge trajectories explored in [20]. This possibility is "opposite" to the broad tensor glueball discussed above (see also the discussion in the end of [20] and Refs. therein). This narrow resonance is in line with the interpretation of a narrow glueball [35]. (we also refer to the note of Doser in [1] on $\left.f_{J}(2220)\right)$.

The branching ratio $\Gamma_{\pi \pi} / \Gamma_{\bar{K} K}=1.0 \pm 0.5$ [1] is compatible with (18). The absence of a $\gamma \gamma$-signal is also in line with a narrow gluonic state. If the glueball is broad, also its vector-vector decay modes are expected to be broad. Therefore in virtue of vector-meson dominance, the two-photon signal is also expected to be large. The general idea that the two-photon signal should be small for a glueball is indeed valid only for a narrow glueball. However, the $\eta \eta^{\prime}$ mode (zero according to the leading-order results expressed in (18)) has been seen, while the $\eta \eta$ mode has not. More precise branching ratios are needed to analyze $f_{J}(2220)$ quantitatively, but this resonance has some intriguing characteristics to be considered as a tensor-glueball candidate.

The properties discussed up to now hold for an unmixed tensor glueball. When considering $f_{J}(2220)$ as a glueball candidate mixing is therefore neglected. In the pseudoscalar meson sector the physical states $\eta$ and $\eta^{\prime}$ are close to octet and singlet states, i.e. far from $\bar{n} n$ and $\bar{s} s$, a fact notoriously connected with the $U_{A}(1)$-QCD anomaly. A strong mixing among $\bar{n} n$ and $\bar{s} s$ is generated, possibly by instantons. A mixing with a pseudoscalar glueball would then be expected to be large, if the $J^{P C}=0^{-+}$glueball had a mass in the energy sector below $1 \mathrm{GeV}$ (but lattice places it higher than $2 \mathrm{GeV}$ ).

The scalar sector is more controversial: a strong glueball-quarkonia mixing is the starting point of the phenomenological works $\mathbf{7},[26,36,37$ and has also been verified by lattice simulation [38, 41]. A strong $\bar{n} n-\bar{s} s$ quarkonia mixing (possibly driven by instantons [42]) has been considered in [25, 26]. In the NJL model (43] and Refs. therein) the $U_{A}(1)$ anomaly is introduced by the t' Hooft interaction term, which affects the pseudoscalar and the scalar mesonic sectors. A gluonic interaction seems therefore enhanced for the $J^{P C}=0^{-+}$and $J^{P C}=0^{++}$states, although in the scalar sector further confirmation is needed. On the other hand, no evidence of enhanced $\bar{n} n-\bar{s} s$ quarkonia mixing is found in other nonets [32]; according to [20], this fact holds also for the excited tensor mesons. The physical states are close to the bare light quark configurations $\bar{n} n$ and $\bar{s} s$. Following this observation, one can argue that a glueball would not mix strongly in this case (this point of view is not accepted by [8], where the state $f_{J}(2220)$ is studied but is considered as a broad resonance). It is important to stress that such a reasoning is qualitative and needs quantitative theoretical analysis, while at the same time a better experimental understanding of $f_{J}(2220)$ is required.

\section{CONCLUSIONS}

In this paper we first studied the decay properties of the ground-state tensor meson nonet: the starting point has been a chiral approach for the two-pseudoscalar decays, where a fit has been performed to deduce the free parameters of the employed Lagrangian (11). The tree-level results are in good agreement with the experimental data. When defining the chiral tensor-pseudoscalar coupling we splitted the tensor meson octet from the singlet: the singlet decay strength turns out to be slightly larger than the octet one. Such a octet-singlet separation is necessary to obtain acceptable results for the fit because of the precision of the experimental data. As a result of the fit the tensor mixing angle and the two-photon decay ratios have been deduced and are in agreement with other approaches and with data.

We then turned our attention to the pseudoscalar-vector and pseudoscalar-photon decay modes for tensor mesons, where the simplest coupling respecting $C P T$-invariance has been considered. The corresponding theoretical results are in good agreement with data.

As a further step of our study on tensor states we considered the tensor glueball, described by a flavor-blind composite field with an independent coupling to two pseudoscalar mesons (the approach is analogous to the analysis in the scalar sector performed in 25, 26 )). The two-pseudoscalar ratios are then presented, where the mass of 2.2 $\mathrm{GeV}$ as obtained on the lattice, has been used. The two-vector decay ratios are then also analyzed. The full strength for the tensor glueball decays is unknown and represents the most interesting and debated issue for glueball decays (not only in the tensor sector). A narrow tensor glueball is discussed in [35], while a broad tensor glueball in [20].

We discussed the narrow resonance $f_{J}(2220)(\Gamma \sim 30 \mathrm{MeV})$ as a possible unmixed tensor-glueball candidate; however, the total spin and the nature of this state are still controversial [1, 8, 20]. The possibility that glueball- 
mixing is small in the tensor sector has been discussed, but further and more quantitative studies are needed in this direction.

\section{Acknowledgments}

This work was supported by the by the DFG under contracts FA67/25-3 and GRK683. This research is also part of the EU Integrated Infrastructure Initiative Hadron physics project under contract number RII3-CT-2004-506078 and President grant of Russia "Scientific Schools" No. 1743.2003.

\section{APPENDIX A: EXPRESSIONS FOR THE DECAY WIDTHS}

The generic two-pseudoscalar decay expression of a tensor state reads (see also Refs. [3, 6, 16]):

$$
\Gamma_{T P_{1} P_{2}}=\alpha_{T P_{1} P_{2}} \frac{P_{T P_{1} P_{2}}^{5}}{60 \pi M_{T}^{2}} g_{T P_{1} P_{2}}^{2}
$$

where $P_{T P_{1} P_{2}}=\lambda^{1 / 2}\left(M_{T}^{2}, M_{P_{1}}^{2}, M_{P_{2}}^{2}\right) / 2 M_{T}$ is the three-momentum of the final states (pseudoscalar mesons) in the rest frame of the decaying initial state (tensor meson) and $\lambda(x, y, z)=x^{2}+y^{2}+z^{2}-2 x y-2 x z-2 y z$ is the Källen triangle function. The $\alpha_{T P_{1} P_{2}}$ takes into account the average over spin of the initial state and the sum over final isospin states with averaging over initial isospin states (symmetry factors included), $g_{T P_{1} P_{2}}$ is the effective $T P_{1} P_{2}$ coupling constant which is defined for different modes as:

$$
\begin{aligned}
\mathcal{L}_{T P_{1} P_{2}} & =\left(g_{f_{2} \pi \pi} f_{2}^{\mu \nu}+g_{f_{2}^{\prime} \pi \pi} f_{2}^{\prime \mu \nu}\right) \partial_{\mu} \vec{\pi} \partial_{\nu} \vec{\pi}+\left(g_{f_{2} \bar{K} K} f_{2}^{\mu \nu}+g_{f_{2}^{\prime} \bar{K} K} f_{2}^{\prime \mu \nu}\right) \partial_{\mu} K^{\dagger} \partial_{\nu} K \\
& +\left(g_{f_{2} \eta \eta} f_{2}^{\mu \nu}+g_{f_{2}^{\prime} \eta \eta} f_{2}^{\prime \mu \nu}\right) \partial_{\mu} \eta \partial_{\nu} \eta+\frac{g_{a_{2} \bar{K} K}}{\sqrt{2}} \partial_{\mu} K^{\dagger} \vec{a}_{2}^{\mu \nu} \vec{\tau} \partial_{\nu} K+\vec{a}_{2}^{\mu \nu} \partial_{\mu} \vec{\pi}\left(g_{a_{2} \pi \eta} \partial_{\nu} \eta+g_{a_{2} \pi \eta^{\prime}} \partial_{\nu} \eta^{\prime}\right) \\
& +\frac{g_{K_{2}^{*} \pi K}}{\sqrt{2}}\left(K_{2}^{* \mu \nu \dagger} \partial_{\mu} \vec{\pi} \vec{\tau} \partial_{\nu} K+\text { h.c. }\right)+g_{K_{2}^{*} \eta K}\left(K_{2}^{* \mu \nu \dagger} \partial_{\mu} K \partial_{\nu} \eta+\text { h.c. }\right)
\end{aligned}
$$

where $\vec{\pi}$ and $\vec{a}_{2}$ are the triplets of pions and tensor $a_{2}$ mesons, $K=\left(K^{+}, K^{0}\right)$ and $K^{\dagger}=\left(K^{-}, \bar{K}^{0}\right)$ are the doublets of kaons, $K_{2}^{*}=\left(K_{2}^{*+}, K_{2}^{* 0}\right)$ and $K_{2}^{* \dagger}=\left(K_{2}^{*-}, \bar{K}_{2}^{* 0}\right)$ are the doublets of tensor $K_{2}^{*}$ mesons.

The results for the parameters involving in Eq. (A1) are reported in Table 3. We introduce the notations: $\tilde{g}_{T P_{1} P_{2}}=$ $g_{T P_{1} P_{2}} / c_{T P P}^{8}$ is the coupling constant scaled by $c_{T P P}^{8}$, the parameter $y=c_{T P P}^{0} / c_{T P P}^{8}$ is the ratio of the singlet and octet couplings, $z_{P}=\left(1+3 \cos 2 \delta_{P}\right) / 2$, where $\delta_{P}=\theta_{P}-\theta_{P}^{I}$ and $\theta_{P}^{I}$ is the ideal mixing angle with sin $\theta_{P}^{I}=1 / \sqrt{3}$.

Table 3. $T \rightarrow P P$ coefficients.

\begin{tabular}{|c|l|c|c|}
\hline$T$ & $P_{1} P_{2}$ & $\alpha_{T P_{1} P_{2}}$ & $\tilde{g}_{T P_{1} P_{2}}$ \\
\hline$f_{2}(1270)$ & $\pi \pi$ & 6 & $\frac{y}{\sqrt{3}} \cos \theta_{T}+\frac{1}{\sqrt{6}} \sin \theta_{T}$ \\
\hline$f_{2}(1270)$ & $\bar{K} K$ & 8 & $\frac{y}{\sqrt{3}} \cos \theta_{T}-\frac{1}{2 \sqrt{6}} \sin \theta_{T}$ \\
\hline$f_{2}(1270)$ & $\eta \eta$ & 2 & $\frac{y}{\sqrt{3}} \cos \theta_{T}-\frac{1}{\sqrt{6}} \sin \theta_{T} z_{P}$ \\
\hline$f_{2}^{\prime}(1525)$ & $\pi \pi$ & 6 & $-\frac{y}{\sqrt{3}} \sin \theta_{T}+\frac{1}{\sqrt{6}} \cos \theta_{T}$ \\
\hline$f_{2}^{\prime}(1525)$ & $\bar{K} K$ & 8 & $-\frac{y}{\sqrt{3}} \sin \theta_{T}-\frac{1}{2 \sqrt{6}} \cos \theta_{T}$ \\
\hline$f_{2}^{\prime}(1525)$ & $\eta \eta$ & 2 & $-\frac{y}{\sqrt{3}} \sin \theta_{T}-\frac{1}{\sqrt{6}} \cos \theta_{T} z_{P}$ \\
\hline$a_{2}(1320)$ & $\bar{K} K$ & 1 & 1 \\
\hline$a_{2}(1320)$ & $\pi \eta$ & 1 & $-\sqrt{2} \sin \delta_{P}$ \\
\hline$a_{2}(1320)$ & $\pi \eta^{\prime}$ & 1 & $\sqrt{2} \cos \delta_{P}$ \\
\hline$K_{2}(1430)$ & $\pi K$ & $3 / 2$ & 1 \\
\hline$K_{2}(1430)$ & $\eta K$ & 1 & $-\frac{1}{\sqrt{6}}\left(\cos \theta_{P}+2 \sqrt{2} \sin \theta_{P}\right)$ \\
\hline
\end{tabular}


The expression for the $P V$ decay width reads $[6]$ :

$$
\Gamma_{T P V}=\alpha_{T P V} \frac{P_{T P V}^{5}}{10 \pi} g_{T P V}^{2},
$$

where $\alpha_{T P V}$ and $\tilde{g}_{T P V}=g_{T P V} / c_{T P V}$ are given in Table 4. The effective couplings $g_{T P V}$ are defined as:

$$
\begin{aligned}
\mathcal{L}_{T V P} & =\frac{g_{f_{2}^{\prime} K K^{*}}}{\sqrt{2}}\left(f_{2}^{\prime[\mu \nu] \alpha} i \partial_{\alpha} K^{\dagger} \tilde{K}_{\mu \nu}^{*}+\text { h.c. }\right) \\
& +\frac{g_{a_{2} \rho \pi}}{\sqrt{2}} \vec{a}_{2}^{[\mu \nu] \alpha} \cdot\left[\partial_{\alpha} \vec{\pi} \times \tilde{\vec{\rho}}_{\mu \nu}\right]+\frac{g_{a_{2} K K^{*}}}{\sqrt{2}}\left(i \partial_{\alpha} K^{\dagger} \vec{a}_{2}^{[\mu \nu] \alpha} \vec{\tau} \tilde{K}_{\mu \nu}^{*}+\text { h.c. }\right) \\
& +\frac{1}{\sqrt{2}} \tilde{K}_{\mu \nu}^{* \dagger}\left[g_{K_{2}^{*} \pi K^{*}} i \partial_{\alpha} \vec{\pi} \vec{\tau}+g_{K_{2}^{*} \eta K^{*}} i \partial_{\alpha} \eta\right] K_{2}^{*[\mu \nu] \alpha}+\text { h.c. } \\
& +\frac{1}{\sqrt{2}} K_{2}^{*[\mu \nu] \alpha \dagger}\left[g_{K_{2}^{*} K \rho} \tilde{\vec{\rho}}_{\mu \nu} \vec{\tau}+g_{K_{2}^{*} K \omega} \tilde{\omega}_{\mu \nu}\right] i \partial_{\alpha} K+\text { h.c. }
\end{aligned}
$$

Table 4. $T \rightarrow P V$ coefficients.

\begin{tabular}{|c|c|c|c|}
\hline$T$ & $P V$ & $\alpha_{T P V}$ & $\tilde{g}_{T P V}$ \\
\hline$f_{2}^{\prime}(1525)$ & $K K^{*}$ & 4 & $\sqrt{3} \cos \theta_{T}$ \\
\hline$a_{2}(1320)$ & $\pi \rho$ & 2 & 2 \\
\hline$a_{2}(1320)$ & $K K^{*}$ & 2 & 1 \\
\hline$K_{2}(1430)$ & $\pi K^{*}$ & 3 & 1 \\
\hline$K_{2}(1430)$ & $\eta K^{*}$ & 2 & $\sqrt{3} \cos \theta_{P}$ \\
\hline$K_{2}(1430)$ & $K \rho$ & 3 & 1 \\
\hline$K_{2}(1430)$ & $K \omega$ & 2 & $\sqrt{3} \sin \theta_{V}$ \\
\hline
\end{tabular}

The $T \rightarrow \gamma \gamma$ decay width reads (see also Refs. [3, 16]):

$$
\Gamma_{T \gamma \gamma}=\frac{\alpha}{20} M_{T}^{3} g_{T \gamma \gamma}^{2},
$$

where $\alpha=e^{2} / 4 \pi=1 / 137$. The couplings $\tilde{g}_{T \gamma \gamma}=g_{T \gamma \gamma} / c_{T \gamma \gamma}$ are given in Table 5 .

Table 5. $T \rightarrow \gamma \gamma$ coefficients.

\begin{tabular}{|c|c|}
\hline$T$ & $\tilde{g}_{T \gamma \gamma}$ \\
\hline$f_{2}(1270)$ & $\frac{4}{3 \sqrt{6}}\left(2 \sqrt{2} \cos \theta_{T}+\sin \theta_{T}\right)$ \\
\hline$f_{2}^{\prime}(1525)$ & $\frac{4}{3 \sqrt{6}}\left(-2 \sqrt{2} \sin \theta_{T}+\cos \theta_{T}\right)$ \\
\hline$a_{2}^{0}(1320)$ & $\frac{4}{3 \sqrt{2}}$ \\
\hline
\end{tabular}

The $T \rightarrow P \gamma$ decay width reads:

$$
\Gamma_{T P \gamma}=\frac{2}{5} \alpha P_{T P \gamma}^{5} g_{T P \gamma}^{2}
$$


where $P_{T P \gamma}=\left(M_{T}^{2}-M_{P}^{2}\right) / 2 M_{T}$ is the three-momentum of final states, $g_{T P \gamma}$ is the coupling constant from the tree-level Lagrangian

$$
\mathcal{L}_{T P \gamma}=\tilde{F}_{\mu \nu}\left\{g_{a_{2} \pi \gamma} a_{2}^{-[\mu \nu] \alpha} i \partial_{\alpha} \pi^{+}+g_{K_{2}^{*} K \gamma} K_{2}^{*-[\mu \nu] \alpha} i \partial_{\alpha} K^{+}\right\}+\text {h.c. }
$$

The factors $\alpha_{T P \gamma}$ and scaled couplings $\tilde{g}_{T P \gamma}=g_{T P \gamma} / c_{T P \gamma}$ are given in Table 6 .

Table 6. $T \rightarrow P \gamma$ coefficients.

\begin{tabular}{|l|l|c|}
\hline \multicolumn{1}{|c|}{$T$} & $P$ & $\tilde{g}_{T P \gamma}$ \\
\hline$a_{2}^{ \pm}(1320)$ & $\pi^{ \pm}$ & 1 \\
\hline$K_{2}^{* \pm}(1430)$ & $K^{ \pm}$ & 1 \\
\hline
\end{tabular}

The decay width of the tensor-glueball $G$ into $P_{1} P_{2}$ pair reads

$$
\Gamma_{G P_{1} P_{2}}=\alpha_{G P_{1} P_{2}} \frac{P_{G P_{1} P_{2}}^{5}}{60 \pi M_{G}^{2}} g_{G P_{1} P_{2}}^{2}
$$

where the corresponding parameters $\alpha_{G P_{1} P_{2}}$ and the coupling constants $\tilde{g}_{G P_{1} P_{2}}=g_{G P_{1} P_{2}} / c_{G P_{1} P_{2}}$ are given in Table 7 . The couplings $g_{G P_{1} P_{2}}$ arise from the tree-level Lagrangian

$$
\mathcal{L}_{G P_{1} P_{2}}=G^{\mu \nu}\left\{g_{G \pi \pi} \partial_{\mu} \vec{\pi} \partial_{\nu} \vec{\pi}+2 g_{G \bar{K} K} \partial_{\mu} K^{\dagger} \partial_{\nu} K+g_{G \eta \eta} \partial_{\mu} \eta \partial_{\nu} \eta+g_{G \eta^{\prime} \eta^{\prime}} \partial_{\mu} \eta^{\prime} \partial_{\nu} \eta^{\prime}\right\}
$$

are, as expected, equal to each other for all the modes, apart from the forbidden $\eta \eta^{\prime}$ one.

Table 7. $G \rightarrow P_{1} P_{2}$ coefficients.

\begin{tabular}{|l|c|c|}
\hline$P_{1} P_{2}$ & $\alpha_{G P_{1} P_{2}}$ & $\tilde{g}_{G P_{1} P_{2}}$ \\
\hline$\pi \pi$ & 6 & 1 \\
\hline $\bar{K} K$ & 8 & 1 \\
\hline$\eta \eta$ & 2 & 1 \\
\hline$\eta^{\prime} \eta^{\prime}$ & 2 & 1 \\
\hline
\end{tabular}

The decay rate of the glueball into two vectors following from the non-derivative coupling (see Eqs. (17) and (21)) reads:

$$
\Gamma_{G V_{1} V_{2}}=\alpha_{G V_{1} V_{2}} \frac{P_{G V_{1} V_{2}}}{8 \pi M_{G}^{2}} F_{G V_{1} V_{2}} g_{G V_{1} V_{2}}^{2}
$$

where

$$
F_{G V_{1} V_{2}}=1+\frac{P_{G V_{1} V_{2}}^{2}}{3}\left(\frac{1}{M_{V_{1}}^{2}}+\frac{1}{M_{V_{2}}^{2}}\right)+\frac{2}{15} \frac{P_{G V_{1} V_{2}}^{4}}{M_{V_{1}}^{2} M_{V_{2}}^{2}} .
$$

In the case of derivative coupling, the function $F_{G V_{1} V_{2}}$ changes (see Ref. [3]) but the factors $\alpha_{G V_{1} V_{2}}$ and $g_{G V_{1} V_{2}}$ do not. As it was mentioned before we consider two specific scenarios: i) the case of $U(3)$ symmetry when the octet $\left(c_{G V V}^{8}\right)$ and singlet $\left(c_{G V V}^{0}\right)$ couplings degenerate $c_{G V V}^{8}=c_{G V V}^{0}=c_{G V V}$; ii) the case of the broken $U(3)$ symmetry to $S U(3)$ one. For convenience we put $c_{G V V}^{8}=c_{G V V}$ and introduce the breaking parameter $y_{G V V}=c_{G V V}^{0} / c_{G V V}^{8}$ which is equal to one at large $N_{c}$ limit (or in case of the $U(3)$ invariance). The couplings $g_{G V_{1} V_{2}}$ arise from the three-level Lagrangian

$$
\mathcal{L}_{G V_{1} V_{2}}=G^{\mu \nu}\left\{g_{G \rho \rho} \vec{\rho}_{\mu} \vec{\rho}_{\nu}+2 g_{G \bar{K}^{*} K^{*}} K_{\mu}^{* \dagger} K_{\nu}^{*}+g_{G \omega \omega} \omega_{\mu} \omega_{\nu}+g_{G \omega \phi} \omega_{\mu} \phi_{\nu}+g_{G \phi \phi} \phi_{\mu} \phi_{\nu}\right\} .
$$


The parameters occurring in Eq. A10 are given in Table 8. Again we rescale $g_{G V_{1} V_{2}}$ by the coupling $c_{G V V}^{8}$ as $\tilde{g}_{G V_{1} V_{2}}=g_{G V_{1} V_{2}} / c_{G V V}^{8}$. In the last two columns of Table 8 we present the results for the couplings $\tilde{g}_{G V_{1} V_{2}}$ for the $U(3)$ symmetric case and for the case of the broken $U(3)$ symmetry.

Table 8: $G \rightarrow V V$ coefficients

\begin{tabular}{|c|c|c|c|}
\hline$V_{1} V_{2}$ & $\alpha_{G V_{1} V_{2}}$ & $\begin{array}{c}\tilde{g}_{G V_{1} V_{2}} \\
{\left[y_{G V V}=1\right]}\end{array}$ & $\begin{array}{c}\tilde{g}_{G V_{1} V_{2}} \\
{\left[\text { any } y_{G V V}\right]}\end{array}$ \\
\hline$\rho \rho$ & 6 & 1 & 1 \\
\hline $\bar{K}^{*} K^{*}$ & 8 & 1 & 1 \\
\hline$\omega \omega$ & 2 & 1 & $\cos ^{2} \theta_{V} y_{G V V}+\sin ^{2} \theta_{V}$ \\
\hline$\omega \phi$ & 1 & 0 & $\sin 2 \theta_{V}\left(1-y_{G V V}\right)$ \\
\hline$\phi \phi$ & 2 & 1 & $\sin ^{2} \theta_{V} y_{G V V}+\cos ^{2} \theta_{V}$ \\
\hline
\end{tabular}


[1] S. Eidelman et al. [Particle Data Group Collaboration], Phys. Lett. B 592, 1 (2004).

[2] B. Renner, Nucl. Phys. B 30, 634 (1971).

[3] M. Suzuki, Phys. Rev. D 47, 1043 (1993).

[4] Y. S. Oh and T. S. H. Lee, Phys. Rev. C 69, 025201 (2004) arXiv:nucl-th/0306033.

[5] S. R. Cotanch and R. A. Williams, Phys. Lett. B 621, 269 (2005) arXiv:nucl-th/0505074.

[6] C. E. Lee and J. F. Min, Chin. J. Phys. 16, 245 (1978) [Print-78-0462 (CHENG KUNG)].

[7] C. Amsler and F. E. Close, Phys. Rev. D 53, 295 (1996) arXiv:hep-ph/9507326.

[8] L. Burakovsky and P. R. Page, Eur. Phys. J. C 12, 489 (2000) arXiv:hep-ph/9906282.

[9] D. M. Li, H. Yu and Q. X. Shen, J. Phys. G 27, 807 (2001) arXiv:hep-ph/0010342.

[10] W. S. Carvalho, A. S. de Castro and A. C. B. Antunes, Eur. Phys. J. C 17, 173 (2000) arXiv:hep-ph/0005193.

[11] A. Lahiri, M. Mukherjee and V. P. Gautam, Prog. Theor. Phys. 63, 698 (1980).

[12] M. Teper, Phys. Lett. B 185, 121 (1987); T. A. DeGrand, Phys. Rev. D 36, 3522 (1987); S. A. Chin, C. Long and D. Robson, Phys. Rev. Lett. 60, 1467 (1988).

[13] E. Bagan and S. Narison, Phys. Lett. B 214, 451 (1988); E. Bagan, A. Bramon and S. Narison, Phys. Lett. B 196, 203 (1987).

[14] C. Gobbi, F. Iachello and D. Kusnezov, Phys. Rev. D 50, 2048 (1994) arXiv:hep-ph/9310250.

[15] E. S. Ackleh, T. Barnes and E. S. Swanson, Phys. Rev. D 54, 6811 (1996) arXiv:hep-ph/9604355.

[16] S. Bellucci, J. Gasser and M. E. Sainio, Nucl. Phys. B 423, 80 (1994) [Erratum-ibid. B 431 (1994) 413] arXiv:hep-ph/9401206.

[17] J. F. Donoghue, C. Ramirez and G. Valencia, Phys. Rev. D 39, 1947 (1989).

[18] D. Toublan, Phys. Rev. D 53, 6602 (1996) [Erratum-ibid. D 57, 4495 (1998)] arXiv:hep-ph/9509217.

[19] C. K. Chow and S. J. Rey, JHEP 9805, 010 (1998) arXiv:hep-ph/9708355.

[20] V. V. Anisovich, JETP Lett. 80, 715 (2004) [Pisma Zh. Eksp. Teor. Fiz. 80, 845 (2004)] arXiv:hep-ph/0412093; V. V. Anisovich, M. A. Matveev, J. Nyiri and A. V. Sarantsev, arXiv:hep-ph/0506133

[21] Y. S. Surovtsev, R. Kaminski, D. Krupa and M. Nagy, Int. J. Mod. Phys. A 20, 561 (2005).

[22] E. Katz, A. Lewandowski and M. D. Schwartz, arXiv:hep-ph/0510388

[23] G. Ecker, J. Gasser, H. Leutwyler, A. Pich and E. de Rafael, Phys. Lett. B 223, 425 (1989).

[24] C. Morningstar and M. J. Peardon, AIP Conf. Proc. 688, 220 (2004) arXiv:nucl-th/0309068.

[25] F. Giacosa, T. Gutsche, V. E. Lyubovitskij and A. Faessler, Phys. Lett. B 622, 277 (2005) arXiv:hep-ph/0504033.

[26] F. Giacosa, T. Gutsche, V. E. Lyubovitskij and A. Faessler, Phys. Rev. D 72, 094006 (2005) arXiv:hep-ph/0509247.

[27] L. Roca, J. E. Palomar and E. Oset, Phys. Rev. D 70, 094006 (2004) arXiv:hep-ph/0306188.

[28] S. Weinberg, Physica A 96, 327 (1979).

[29] J. Gasser and H. Leutwyler, Annals Phys. 158, 142 (1984); Nucl. Phys. B 250, 465 (1985).

[30] G. Ecker, J. Gasser, A. Pich and E. de Rafael, Nucl. Phys. B 321, 311 (1989); G. Ecker, J. Gasser, H. Leutwyler, A. Pich and E. de Rafael, Phys. Lett. B 223, 425 (1989).

[31] E. P. Venugopal and B. R. Holstein, Phys. Rev. D 57, 4397 (1998) arXiv:hep-ph/9710382.

[32] V. Cirigliano, G. Ecker, H. Neufeld and A. Pich, JHEP 0306, 012 (2003) arXiv:hep-ph/0305311.

[33] B. Borasoy and U. G. Meissner, Int. J. Mod. Phys. A 11, 5183 (1996) arXiv:hep-ph/9511320; B. Kubis and U. G. Meissner, Nucl. Phys. A 679, 698 (2001) arXiv:hep-ph/0007056.

[34] P. Minkowski and W. Ochs, Nucl. Phys. Proc. Suppl. 121, 123 (2003) arXiv:hep-ph/0209225; P. Minkowski and W. Ochs, arXiv:hep-ph/9905250

[35] C. Amsler and N. A. Tornqvist, Phys. Rept. 389, 61 (2004).

[36] M. Strohmeier-Presicek, T. Gutsche, R. Vinh Mau and A. Faessler, Phys. Rev. D 60 (1999) 054010 arXiv:hep-ph/9904461.

[37] F. E. Close and A. Kirk, Eur. Phys. J. C 21, 531 (2001) arXiv:hep-ph/0103173.

[38] W. J. Lee and D. Weingarten, Phys. Rev. D 61, 014015 (2000) arXiv:hep-lat/9910008; J. Sexton, A. Vaccarino and D. Weingarten, Nucl. Phys. Proc. Suppl. 47, 128 (1996) arXiv:hep-lat/9602022 .

[39] F. Giacosa, T. Gutsche and A. Faessler, Phys. Rev. C 71, 025202 (2005) arXiv:hep-ph/0408085].

[40] V. V. Anisovich, M. A. Matveev, J. Nyiri and A. V. Sarantsev, arXiv:hep-ph/0501003 V. V. Anisovich and A. V. Sarantsev, JETP Lett. 81, 417 (2005) [Pisma Zh. Eksp. Teor. Fiz. 81, 531 (2005)] arXiv:hep-ph/0504106].

[41] C. McNeile and C. Michael [UKQCD Collaboration], Phys. Rev. D 63, 114503 (2001) arXiv:hep-lat/0010019.

[42] E. Klempt, arXiv:hep-ex/0101031

[43] T. Hatsuda and T. Kunihiro, Phys. Rept. 247, 221 (1994) arXiv:hep-ph/9401310. 